\title{
Ensayo sobre una aplicación de un ciclo de mejora en el aula. Acercamiento, destrucción y dilatación del fenómeno ético en Aristóteles
}

\section{Essay on an application of an Improvement Cycle in Classroom. Approach, destruction and expansion of the ethical phenomenon in Aristotle}

JUAN JosÉ GARRIDo PERIÑÁN

ORCID: https://orcid.org/0000-0002-7586-7579

Universidad de Sevilla

Departamento de Estética e

Historia de la Filosofía

iigarper@us.es

DOI: http://dx.doi.org/10.12795/9788447231003.030

Pp.: 614-629 


\section{Introducción}

Después de la realización de un Ciclo de Mejora en el Aula (CIMA), realizado hace ya varios meses e interrumpido a causa de la pandemia COVID-19, me propongo llevar a cabo un segundo ciclo, de cara a la presentación de esta publicación y en una titulación diferente y con un nivel de especialización más avanzado, propio de los estudios de posgrado. La asignatura se intitula: "El legado histórico de la antigüedad en el mundo moderno", impartida dentro de la titulación "Máster en Filosofía y Cultura Moderna", en el curso 2020/21. Al coincidir el inicio de la asignatura, justo con la aplicación de este CIMA, los aspectos que se abordarán para activar el proceso de enseñanza anhelado deben ser propedéuticos, que sirvan para que el discente, antes de entrar por asi decir "en faena", sepa orientarse en las problemáticas primarias, concernientes a la cuestión del acceso, o sea:

- ¿cómo empezar a abordar un problema como el de la ética?

- ¿somos seres éticos por naturaleza?

- ¿está la ética asegurada, de antemano, en todas nuestras acciones?

- ¿Si la ética no estuviera asegurada, sería menester una activación?

Como se espera mostrar a lo largo del trabajo, la realización del CIMA fue todo un éxito, aunque los motivos de tal estado de éxito son relativizados a causa del carácter propedéutico y provisorio de la tarea docente llevada a cabo. Asimismo, se incorporan valiéndonos de parte de la tradición fenomenológica-hermenéutica, ámbito de especialización dentro de los estudios filosóficos, de 3 procedimientos intelectuales que vertebrarán el mecanismo mediante el cual se ha ejecutado el proceso de enseñanza, a saber: procedimiento intelectual de contraste, procedimiento intelectual destructivo y procedimiento intelectual

Ciclos de Mejora en el Aula (2020). Experiencias de Innovación Docente de la US Esta obra se distribuye con la licencia Creative Commons 
expansivo o dilatador. Y todo ello de cara a que el discente pueda desarrollar sus capacidades ínsitas en torno a un comportamiento despierto y crítico.

\section{Diseño del Ciclo de Mejora en el Aula: un modelo filosófico-existencial sobre la cuestión ética.}

El CIMA se proyectó para 8 horas docentes. Estas horas estuvieron distribuidas en dos semanas, a 4 horas por cada semana. La docencia se impartió los martes y los jueves de 18:00 a 20:00 horas, de manera totalmente presencial. El contenido impartido fue, en consecuencia, el primero, que lleva por título: “Qué es la ética? ¿Cómo es la ética? ¿Quién es la ética?” Ante esto debo advertir lo que sigue: como tema introductorio no se trataría de responder a las tres preguntas recientemente mentadas, sino tan solo de hendir un horizonte que nos prepare para responder con pertinencia tales preguntas. Esto significa que este CIMA no pudo estar orientado a la transmisión neta de un contenido concreto, tampoco esta vicisitud se tomó como una preocupación directa sobre qué contenido impartir; más bien, el problema genuino estribó en la capacidad de activar en los discentes una problemática -la cuestión ética-, dilatando el ámbito de interrogación humana, y, por último, ajustando tal ámbito dentro una perspectiva existencial. En verdad, si nos fijamos en el título del tema a impartir, este CIMA trató de cómo es la ética, y esto desde una perspectiva muy limitada.

A continuación, voy a compartir el mapa de contenido, estructurado desde estos tres ámbitos interrogativos qué/ cómo/quién es la ética, los cuales actuaron como vectores indispensables en el proceso de aprendizaje

Ciclos de Mejora en el Aula (2020). Experiencias de Innovación Docente de la US Esta obra se distribuye con la licencia Creative Commons 


\section{JUAN JOSÉ GARRIDO PERIÑÁN}

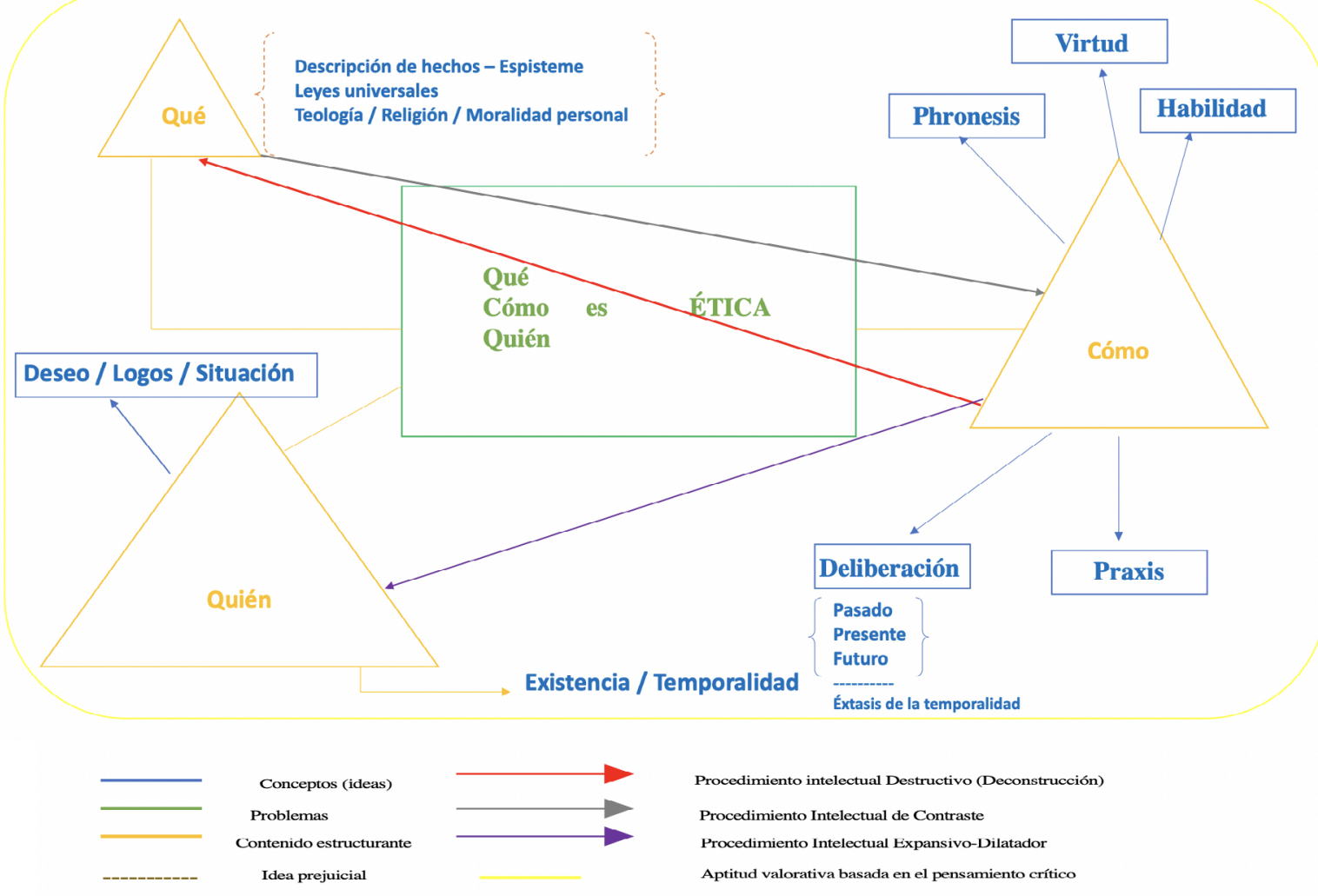

Figura 1. Mapa de contenidos

Ciclos de Mejora en el Aula (2020). Experiencias de Innovación Docente de la US (c) (i) Esta obra se distribuye con la licencia Creative Commons cc) Internacional (CC BY-NC-ND 4.0.) 
Como ya he advertido, en el presente mapa de contenidos apenas hay conocimiento tasado como teórico, aunque parezca lo contrario. Lo coloreado en rojo, signado como conceptos (ideas), son más bien llaves que posibilitan la apertura a la comprensión de otro modo (estructura del cómo en detrimento del qué). En este sentido, durante las 8 horas del CIMA, no se abordaron los conceptos aristotélicos de modo directo, contrastándolos con otras nociones y con la exégesis heredada por la tradición. Esto no se hizo, empero, porque se pensó hacer con posterioridad. De lo que se trata es que los conceptos (ideas) sirvan de indices orientativos. La parte dedicada a problemas, como se ve, encaminada desde 3 preguntas prima facie muy abstractas, fueron desarrolladas al socaire del cuestionario inicial. También, como se observa, me he permitido subdividir los procedimientos intelectuales en tres tipologías, a saber:

\section{- Procedimiento intelectual de contraste (PIC) \\ - Procedimiento intelectual destructivo (PID) \\ - Procedimiento intelectual expansivo (o dilatador) (PIE)}

Aunque todavía debo pensar en ello con más demora y dedicación, de cara a un abordaje teórico más riguroso, mediante PIC miento un procedimiento cognoscitivo de implicación y contraste, la pregunta qué es la ética va a resultar insuficiente y va a dirigirse inexorablemente a la interrogación por la situación (su respectivo cómo) y la incertidumbre inalienable de la decisión deliberativa (phronesis / prudencia). Con PID se trata de la acción de una deconstrucción, es decir, no se anhelaría destruir o erradicar el saber anterior (el qué de la ética), sino de determinarlo en su justa situación. En este sentido la destrucción vendría a resituar el fenómeno de la ética desde su ámbito propio de mostración, que no es la teoría, sino la praxis, las acciones humanas gobernadas por las tensiones entre el deseo y la razón, la avaricia y la prudencia, las cuales no pueden ser regladas al modo en que lo hace la 
ciencia física, por ejemplo, debiendo soportar un principio de indeterminación (incertidumbre, inseguridad) constante. Con PIE defino el consecuente de PID, el horizonte generado por la acción de la destrucción que viene a vincular el fenómeno de la ética con una ontología de la existencia humana basada en su temporalidad vivida, porque la decisión deliberativa se asienta en la estructura ontológica de la temporalidad humana, donde el pasado nunca está detrás, y solo queda un futuro que se abre como instante (situación) que, a su vez, nos demanda siempre ya un tener que decidirse con algo (Heidegger, 2000; Garrido, 2019). Como se echa de ver, en el mapa de contenidos, hay una omisión deliberada en el aspecto de las consideraciones valorativas. No estimo pertinente planificar e introducir en el discente un contenido valorativo a sabiendas, con intencionalidad, pues es algo que dependerá de la biografía de cada discente y del modo en que cada uno asuma su existencia. Esto no significa que rechace de plano una enseñanza de los afectos, sino el método o la manera según la cual un docente deba trabajar por anticipado en generar afectos. Según entiendo, los afectos no deberían generarse en educación, como lo hace, por ejemplo, el marketing o la publicidad, sino en efecto enseñar qué/ cómo asumir lo que nos pasa desde el saber de nuestra disciplina. La filosofía desde la antigua Grecia se ha preocupado sobre esa dimensión humana denominada pathos (afecto, pasión, sufrimiento), y se ha procurado la posibilidad de una enseñanza a través del pathos, el sufrimiento (Mena, 2019). Como se podría intuir, las preguntas-problemas pueden generar sobre los discentes perplejidad, algunos tendrán que luchar contra sus prejuicios religiosos, por ejemplo, o contra sus convicciones morales, etc. Y esto, desde luego, es muy importante para la enseñanza, pero, al menos desde mi parecer, la tarea docente no ha de consistir en determinar qué es lo mejor, correcto o incorrecto. Aquí, en términos de la asignatura, estaríamos tratando de moral, no de ética (exige una deliberación basada en 
la temporalidad afectica-comprensiva de cada individuo; en cambio, la moral sería del orden acusativo-imperativo, como los mandamientos). Así, de este modo, en calidad de actitud y aptitud relativamente valorativa, después de lo manifestado, solo se ha contemplado la "actitud despierta o crítica" como objetivo basal del proceso de enseñanza a favor de los discentes.

A fin de llevar a cabo de manera práctica el mapa de contenido se propuso el siguiente modelo metodológico:

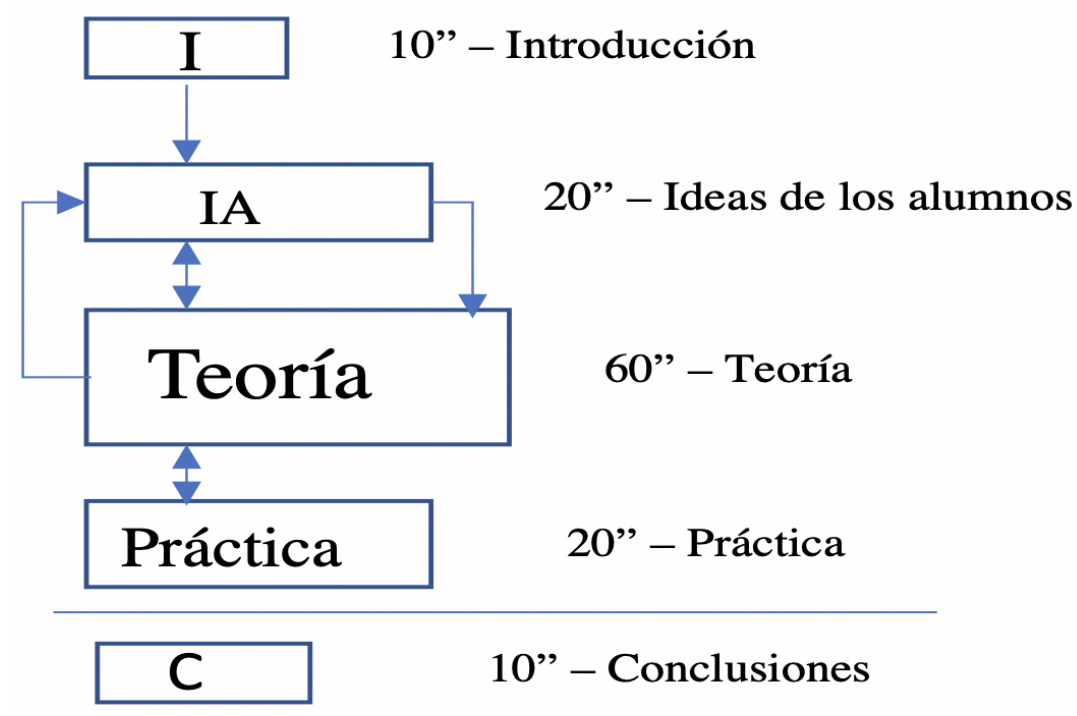

Figura 2. Modelo Metodológico

El CIMA estuvo enfocado, con preeminencia, para dar cumplimiento a los procedimientos intelectuales (PIC/ PID/PIE), mediante el decurso del problema éticf ${ }^{\circ}$, y las cuestiones vectoriales qué/cómo/quién. De este modo, la secuencia de actividades específica para trabajar el mapa de contenidos y dar sentido al modelo metodológico en el aula fue la siguiente:

Clase 1 (2 horas) Aplicación del PIC / QUÉ

10 min - Introducción: se presentan los aspectos básicos del curso.

Ciclos de Mejora en el Aula (2020). Experiencias de Innovación Docente de la US Esta obra se distribuye con la licencia Creative Commons 
20 min - Ideas de los alumnos: se explica y entrega cuestionario inicial 1.

60 min - Teoría: se empieza preguntando a los alumnos sobre lo que han escrito y se aprovecha para abrir la problemática filosófica. Se intenta mostrar la insuficiencia de la tarea de definir qué es la ética, justificando cierta tendencia a la "dogmatización" (Procedimiento PIC).

20 min - Práctica: cada alumno lee un pequeño texto de Aristóteles.

10 min - Conclusiones: en verdad, no se concluye nada, sino que se dejan abiertos muchos interrogantes con el objeto de darles respuesta en la siguiente clase.

\section{Clase 2 (2 horas) Aplicación del PID / CÓMO}

10 min - Introducción: se recuperan las preguntas nucleares que nos dejaron perplejos (inquietos, aburridos, extasiados, hastiados...) en la sesión anterior.

20 min - Ideas de los alumnos: sigo aprovechando las preguntas del cuestionario 1. La idea es que problematicen mediante el diálogo si es posible una ciencia de lo que estudia la ética.

60 min - Teoría: desde lo dicho por los discentes, el profesor llevará a cabo el PID. Toda la explicación teórica se empleará para explicar las características particulares de lo que Aristóteles llama ética, la diferenciación con respecto a la moral (qué) y dejará vía libre para que los discentes tengan un acceso filosófico (que no estaba garantizado antes, de partida, siendo posibilitado por lo que he llamado destrucción) al asunto de la ética.

20 min - Práctica: Selecciono a dos alumnos (voy a optar por chico y chica) y les digo que, en 10 minutos para cada uno, y como máximo, defiendan dos posturas antagónicas, a saber:

a) la ética es una ciencia (trata de verdades universales);

b) la ética es un tipo de saber práctico y contingente.

10 min - Conclusiones: participación de todos los alumnos.

Ciclos de Mejora en el Aula (2020). Experiencias de Innovación Docente de la US Esta obra se distribuye con la licencia Creative Commons 


\section{Clase 3 (2 horas) Aplicación del PIE / QUIÉN}

10 min - Introducción: se recuperan las preguntas nucleares que nos dejaron perplejos (inquietos, aburridos, extasiados, hastiados...) en la sesión anterior.

20 min - Ideas de los alumnos: se recuperan aspectos del debate.

60 min - Teoría: vinculo el cómo con el quién; muestro que no hay ética sin existente y que la ética no puede exceder los límites de toda temporalidad; la temporalidad se muestra en la decisión y deliberación.

20 min - Práctica: los alumnos leen unos fragmentos de Heidegger.

10 min - Conclusiones: participación de todos los alumnos.

\section{Clase 4 (2 horas) Aplicación del PIE / QUIÉN}

10 min - Introducción: se recuperan las preguntas nucleares que nos dejaron perplejos (inquietos, aburridos, extasiados, hastiados...) en la sesión anterior.

20 min - Ideas de los alumnos: se recuperan asuntos del texto de la práctica anterior.

60 min - Teoría: se explica que la comprensión de nuestra temporalidad pende la manera en la que, en cada caso, asumimos la posibilidad de la imposibilidad del existir (fenomenología de la finitud-muerte humana)

20 min - Práctica: los alumnos deben de volver a responder las preguntas del cuestionario 1.

10 min - Conclusiones: participación de todos los alumnos.

El modelo pedagógico preferido, atendiendo a las propias limitaciones situacionales del momento, intenta siempre partir del estado medio o interpretación cotidiana sobre el fenómeno a tratar, para luego, mediante el ejercicio de una exposición teórica que no excluye el diálogo y la participación del discente, mostrar las insuficiencias y limitaciones de las posturas adoptadas, muchas veces 
asumidas desde ciertas convicciones incuestionables, pero que necesitan ser espoleadas de cara a posibilitar otro modo de comprenderlas y asumirlas. Luego, esta enseñanza adquirida, puesto a concursar el PIC y el PID, se concretiza en una actividad práctica a modo de consolidación de lo aprendido. Esta consolidación busca ser todavía más clarificada con el abordaje de conclusiones que, presentando un carácter abierto y nada definitivo, sirven a modo de ideas fuerza de cara a futuras lecciones.

Si tomamos en cuenta la necesidad de partir de la situación real mediante la cual un discente se relaciona con lo que va a constituir su objeto de estudio, resulta fundamental insertar un cuestionario inicial como el que se comparte a continuación:

Tabla 1. Cuestionario inicial

Contesta a las siguientes preguntas sin acudir a definiciones eruditas ni prestadas en los libros. Habla desde tu propia experiencia, haciendo valer tu saber cotidiano. Aquí no se busca respuestas correctas, sino respuestas honestas, que salgan de tu existencia.

1. ¿Qué tipo de palabras se te vienen a la cabeza cuando escuchas la palabra "ética"? (Máx. 5).

2. Consideras como "éticas" cuestiones tales como (decir por qué):

a. ¿Cuántas jarras de Cruzcampo (cerveza) me conviene beber?

b. ¿Debe un neurocirujano a punto de realizar una operación beber algo de alcohol?

c. Si estamos de vacaciones ¿es errado beber algún trago (alcohol)?

3. ¿Podría considerarse buen médico alguien que diagnosticase certeramente las causas y efectos de la COVID-19, a pesar de que en tal procedimiento muriesen todos sus pacientes?

4. ¿Considerarías una acción virtuosa aquella que hace de la incontinencia (no beber alcohol) un principio para siempre, absoluto? (Es decir, la acción de no beber nunca, sin atender a la circunstancia)

Este cuestionario pretendió insertar sobre los discentes un vínculo existencial con el tema de la asignatura, haciéndoles ver la intrínseca adherencia que ellos, en tanto existentes, comportan con relación al problema de la ética, 
solo por el mero y nada insignificante hecho de existir, esto es, de tener que realizarse sobre posibilidades y asumir tales posibilidades como propias. Las preguntas que integran el cuestionario, además de partir de una situación básica, propia de la cotidianidad, se articulan siguiendo las preguntas basales del contenido pedagógico en torno al qué, al cómo y al quién es la ética, a saber:

- La pregunta número 1 está emparentada con el qué.

- La pregunta 2, sus sub-preguntas y la pregunta 3 se encuentran relacionadas con el cómo.

- La pregunta 4, en cambio, procura vincularse con el quién.

Mediante este emparejamiento producido entre el cuestionario y el contenido pedagógico no solo se busca dotar de unidad al proceso de enseñanza, sino mostrar en qué sentido el objeto de estudio de la asignatura nace y se nutre de problemáticas reales, urgentes para el desarrollo de la vida humana. El cuestionario, como ya se ha recogido anteriormente, fue pasado a los estudiantes al inicio y al final del proceso, a fin de determinar sus modelos mentales.

\section{Aplicación del Ciclo de Mejora en el Aula: un éxito contenido}

Empezar una asignatura, como ha sucedido en este caso, es siempre emocionante, un momento de incertidumbre en el que el profesor y los discentes se ven por vez primera. La aplicación del CIMA podría haber agravado la incertidumbre, pero valió más como motivación, más cuando en el panorama actual cualquier ejercicio de innovación resulta una rareza. El uso del cuestionario inicial sirvió para que los discentes se enfrentarán por vez primera con ciertas preguntas que, en razón a su contenido, 
les resultaban familiares, pero inapropiadas dentro de un contexto como el universitario. Esto logró, ciertamente, un primer vínculo con la asignatura, pues pronto se vio cierta adhesión a los problemas fundamentales que íbamos a impartir a lo largo de las sesiones. El uso de la explicación magistral, siempre intercalada por preguntas de los alumnos, se centró en potenciar y desarrollar las problemáticas abiertas, desde aspectos concretos y cotidianos, como si es ético beber alcohol antes de una operación quirúrgica, o antes de conducir; también mediante cuestiones que pusieron en juego la cuestión del deseo, en la medida en que se lo consideró un vector imprescindible para hacer posible una acción ética. En este sentido, otro de los aspectos vitales fue recuperar y traer al presente un pensamiento arcaico como el de Aristóteles, mostrándoles a los discentes no solo su actualidad, sino el suelo originario que nutrió su filosofar, siempre en respuesta a problemas y experiencias existenciales, que nos demandan un tener que asumir lo que somos.

Los discentes, atendiendo al efecto de la enseñanza sobre sus propios estados afectivos, algunos mostraban caras de perplejidad; otros, desconcierto, asombro, seriedad, ilusión, indiferencia, etc. Ante tanta gama afectiva, me centré en justificar que el filosofar debe anidar en una experiencia concreta con el objeto de volver lúcida, comprensible la propia experiencia. Solo así la máxima de conócete a ti mismo es posible.

Con normalidad los ejercicios de innovación docente suelen ser bien recibidos. Concibo esta condición debida a la novedad que supone, en un sistema educativo momificado, con cierta señal de decadencia creativa, cada vez más profesionalizado y, en consecuencia, desvitalizado y desarraigado de su mismo suelo. Todos los ejercicios propuestos, desde mi opinión de docente, han funcionado, a saber:

- El cuestionario docente ha servido para ganar un primer vínculo con el contenido de la asignatura. 
- Los ejercicios prácticos, lecturas de textos y debates, han logrado que la participación sea positiva, por cuanto, desde la opinión, se han puesto en liza interpretaciones y comprensiones imprescindibles en el aprendizaje.

- Las explicaciones teóricas han funcionado, pues han logrado despertar interés y participación en los discentes.

El cuestionario inicial, por lo demás, fue respondido por 32 estudiantes, de entre ellos 3 compartieron una respuesta muy erudita, empleando palabras prestadas por otros autores, cuando no se demandaba tal cosa. El cuestionario final presenta resultados asombrosos, por cuanto una media de entre 82 y $95 \%$ hacen ver que han asimilado el proceso de enseñanza. A modo de ejemplo, se exponen los resultados de los cuestionarios inicial y final en relación con la pregunta 4 y el contenido pedagógico que gira en torno a la pregunta quién es la ética:
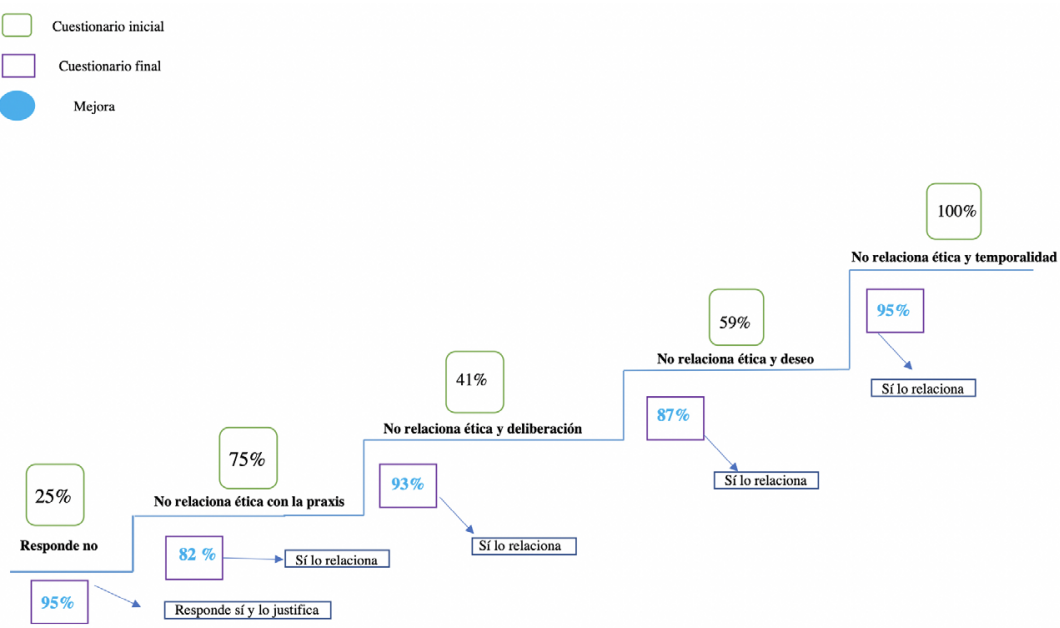

Figura 3. Escalera de aprendizaje del tema quién es la ética

Como se echa de ver el resultado ha sido excelente. Los motivos del éxito pueden deberse al carácter introductorio del CIMA llevado a cabo, donde no era necesario ahondar

Ciclos de Mejora en el Aula (2020). Experiencias de Innovación Docente de la US Esta obra se distribuye con la licencia Creative Commons 
en contenido arduo. Por los resultados del cuestionario final se puede observar de manera palmaria que el proceso de enseñanza ha resultado positivo. En todos los casos propuestos, de estratificación del contenido docente, todos los discentes han mejorado sus resultados logrando una maduración intelectual importante, siendo capaces de apropiarse de lo enseñado. Sobre las demás preguntas que vertebraron el cuestionario docente, se aprecian resultados muy similares, una media de $85 \%$ de todos los discentes mejoran, logrando apropiarse el contenido docente de la asignatura.

\section{Evaluación del CIMA: un camino por seguir de mejora y compromiso}

Creo que, a pesar del éxito de las medidas adoptadas, debería incrementar el trabajo de lecturas, esencial en el aprendizaje de la filosofía. El discente suele congratularse cuando entiende fácilmente un tipo de pensamiento, que por lo general ha costado sudor y lágrimas al autor descifrar, y esto conlleva cierta tendencia a "rebajar" lo pensado por tal autor o autora. Debo insertar mecanismos que lleven a los discentes a un esfuerzo mayor, en vez de presentarlo todo de manera sencilla, sin interferencias. Por las sesiones de formación docente recibidas en el programa FIDOP (Porlán, 2017), sé que hay posibilidades estratégicas a fin de obtener un compromiso mayor de parte del discente, pero el compromiso que busco no es el consecuente de un estado de seducción pedagógica, sino el de un denodado esfuerzo por descifrar, por ejemplo, la interpretación de un determinado filósofo, la cual pudiera servir de óbice para la continuación del aprendizaje, pero que el discente debiera saber responder, sin rendición, a tal desafío. Como se echa de ver, lo que digo conllevaría un nivel de profundización mayor, que ahora mismo no alcanzo, pero sería una meta dentro de mi propia formación como

Ciclos de Mejora en el Aula (2020). Experiencias de Innovación Docente de la US Esta obra se distribuye con la licencia Creative Commons 
docente. Debo decir, por último, que el solo hecho de demorarme en el pensar sobre mi docencia es un fenómeno positivo, pues así uno observa sus propias limitaciones, y puede mejorar posibles errores. Centrar la docencia en el aprendizaje del alumno es imprescindible, siendo el sujeto protagónico de la enseñanza.

Palabras clave: El legado histórico de la antigüedad en el mundo moderno, Máster en Filosofía y Cultura Moderna, docencia universitaria, experimentación docente universitaria, filosofía existencial

Keywords: The historical legacy of antiquity in the modern world, Master in Philosophy and Modern Culture, university teaching, university teaching experimentation, existential philosophy

Ciclos de Mejora en el Aula (2020). Experiencias de Innovación Docente de la US Esta obra se distribuye con la licencia Creative Commons 


\section{Referencias bibliográficas}

Garrido, J. J. (2019). La aportación no-apofántica de la dișposición afectiva y la mismidad del Dasein: análisis fenomenológico a partir del momento estructural seren. Pensamiento: Revista de Investigación e Información Filosófica, 75(285), 887-911.

Heidegger, M. (1993). Ontología: Hermenéutica de la facticidad. Madrid: Alianza Editorial.

Mena, P. (2019). El pathos de la vida y de la existencia. La fenomenología en busca de una ampliación de la razón. Anales del Seminario de Historia la Filosofía, 36(1), 201-220.

Porlán, R. (Coord.) (2017). Enseñanza universitaria. Cómo mejorarla. Madrid: Morata.

Ciclos de Mejora en el Aula (2020). Experiencias de Innovación Docente de la US Esta obra se distribuye con la licencia Creative Commons 